\title{
Nonlinear Harmonic Generation of Coherent Amplification and Self-Amplified Spontaneous Emission
}

\author{
Zhirong Huang ${ }^{1}$, Kwang-Je Kim \\ Advanced Photon Source, Argonne National Laboratory, Argonne, IL 60439, USA
}

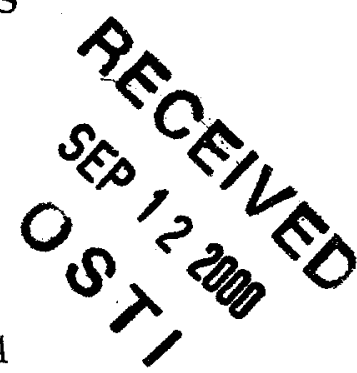

\begin{abstract}
Nonlinear harmonic generation in a uniform planar undulator is analyzed using the three-dimensional Maxwell-Klimontovich equations that include both even and odd harmonic emissions. After a certain stage of exponential growth, the dominant nonlinear harmonic interaction is caused by strong bunching at the fundamental. As a result, gain length, transverse profile, and temporal structure of these harmonic radiations are eventually determined by those of the fundamental. Transversely coherent third-harmonic radiation power is found to approach one percent of the fundamental power level for current high-gain FEL projects, while the power of the second-harmonic radiation is less but still significant for relatively low-energy FEL experiments.
\end{abstract}

Key words: Nonlinear Harmonic generation; Coherent Amplification; Self-amplified spontaneous emission PACS: 41.60.Cr; 42.55.Vc; 42.65.Ky

The submitted manuscript has been created by the University of Chicago as Operator of Argonne National Laboratory ("Argonne") under Contract No. W-31-109-ENG-38 witt. the U.S. Department of Energy. The U.S. Government retains for itself, and others acting on its behalf, a paid-up, nonexclusive. irrevocable worldwide license in said article to reproduce, prepare derivative works, distribute copies to the public, and perform publicly and display publicly, by or on behalf of the Government.

\section{Introduction}

In a high-gain free-electron laser (FEL) employing a planar undulator, a onedimensional (1-D) model [1] and a three-dimensional (3-D) simulation [2] indicate that strong bunching at the fundamental wavelength can drive substantial harmonic bunching and sizable power levels at the harmonic frequencies.

\footnotetext{
${ }^{1}$ Corresponding author. Tel: (630)252-6023; Fax: (630)252-5703; Email: zrh@aps.anl.gov
} 


\section{DISCLAIMER}

This report was prepared as an account of work sponsored by an agency of the United States Government. Neither the United States Government nor any agency thereof, nor any of their employees, make any warranty, express or implied, or assumes any legal liability or responsibility for the accuracy, completeness, or usefulness of any information, apparatus, product, or process disclosed, or represents that its use would not infringe privately owned rights. Reference herein to any specific commercial product, process, or service by trade name, trademark, manufacturer, or otherwise does not necessarily constitute or imply its endorsement, recommendation, or favoring by the United States Government or any agency thereof. The views and opinions of authors expressed herein do not necessarily state or reflect those of the United States Government or any agency thereof. 


\section{DISCLAIMER}

Portions of this document may be illegible in electronic image products. Images are produced from the best available original document. 
A 3-D analysis of harmonic generation in a uniform planar undulator has been given in Ref. [3], for the process of coherent amplification (CA) and self-amplified spontaneous emission (SASE). In this paper, we extend the formalism of Ref. [3] to include the generation of even harmonics and present analytic formulas for computing the second and the third nonlinear harmonic powers as well as the bunching parameters. Explicit calculations based on current high-gain FEL projects show that the power of the transversely coherent third-harmonic radiation can approach one percent of the fundamental power level, while the power of the second-harmonic radiation is less but still significant for relatively low-energy FEL experiments.

\section{Nonlinear Harmonic Generation}

For an electron in a planar undulator (with the undulator parameter $K$ ), the transverse wiggling motion in the $x$ plane is accompanied by a longitudinal oscillation (at twice the transverse frequency $c k_{u}$ ) about the average longitudinal position $c t^{*}$. This figure-eight motion (in the comoving frame) can give rise to harmonic emissions. Let us represent the electric field in the form

$$
\hat{x} \int_{-\infty}^{\infty} \frac{d \nu}{2} E(\nu, \mathbf{x} ; z) e^{i \nu k_{1}(z-c t)}
$$

where $\mathrm{x}=(x, y)$ represents the transverse coordinates, $c k_{1}=\frac{2 \gamma_{0}^{2} c k_{u}}{1+K^{2} / 2}$ is the fundamental resonant frequency, and $|E(\nu)|$ is the field amplitude at frequency $\omega=\nu k_{1} c$.

It is convenient to treat $z$, the distance from the undulator entrance, as the independent variable. and change the dependent coordinate from $t$ to $\theta$ by $\theta(z)=\left(k_{u}+k_{1}\right) z-c k_{1} t^{*}=\left(k_{u}+k_{1}\right) z-c k_{1} t+\xi \sin \left(2 k_{u} z\right)$, where $\xi=K^{-2} /(4+$ 
$\left.2 K^{2}\right)$. The Maxwell equation under the paraxial approximation becomes

$$
\begin{aligned}
\left(\frac{\partial}{\partial z}+\frac{\nabla_{\perp}^{2}}{2 i \nu k_{1}}\right) & E(\nu, \mathbf{x} ; z)=-\frac{e K}{\epsilon_{0} \gamma_{0}} \int_{-\infty}^{\infty} \frac{k_{1} d \theta}{2 \pi} e^{-i \nu \theta} e^{i \nu k_{u} z+i \nu \xi \sin \left(2 k_{u} z\right)} \cos \left(k_{u} z\right) \\
& \times \sum_{j=1}^{N_{e}} \delta\left(x-\frac{K}{\gamma_{0} k_{u}} \sin \left(k_{u} z\right)-x_{j}^{\beta}\right) \delta\left(y-y_{j}^{\beta}\right) \delta\left(\theta-\theta_{j}\right),
\end{aligned}
$$

where $N_{e}$ is the total number of electrons, and $x_{j}^{\beta}$ and $y_{j}^{\beta}$ describe the transverse betatron oscillations. Because the transverse wiggling amplitude is normally smaller than the transverse dimension of the electron beam, we approximate

$$
\delta\left(x-\frac{K}{\gamma_{0} k_{u}} \sin \left(k_{u} z\right)-x_{j}^{\beta}\right) \approx \delta\left(x-x_{j}^{\beta}\right)-\frac{K}{\gamma_{0} k_{u}} \sin \left(k_{u} z\right) \delta^{\prime}\left(x-x_{j}^{\beta}\right),
$$

where $\delta^{\prime}=d \delta /(d x)$. Since the FEL interaction and the betatron oscillation occur on a scale much longer than the fast wiggling motion, we average Eq. (2) over the undulator period $\lambda_{u}$ with the help of the Bessel function expansion

$$
e^{i \nu \xi \sin \left(2 k_{u} z\right)}=\sum_{p=-\infty}^{+\infty} J_{p}(\nu \xi) e^{i 2 p k_{u} z} .
$$

Inserting the first term of Eq. (3) into Eq. (2), we find that the wiggling averaging is nonzero only when $\nu$ is close to an odd integer $h=-(2 p \pm 1)$ [4] and obtain the equation for odd harmonics [3]:

$$
\begin{aligned}
\left(\frac{\partial}{\partial z}+\frac{\nabla_{\perp}^{2}}{2 i h k_{1}}\right) E_{h}\left(\Delta \nu_{h}, \mathbf{x} ; z\right)= & -\frac{e K_{h}}{2 \epsilon_{0} \gamma_{0}} e^{i \Delta \nu_{h} k_{u} z} \int \frac{k_{1} d \theta}{2 \pi} e^{-i \nu \theta} \\
& \times \sum_{j=1}^{N_{e}} \delta\left(\mathbf{x}-\mathbf{x}_{j}^{\beta}\right) \delta\left(\theta-\theta_{j}\right),
\end{aligned}
$$

where $\Delta \nu_{h}=\nu-h \ll 1$ is the frequency detuning and the effective coupling strength is

$$
K_{h}=K(-1)^{(h-1) / 2}\left[J_{(h-1) / 2}(h \xi)-J_{(h+1) / 2}(h \xi)\right], \quad h=1,3,5, \ldots
$$

Inserting the second term of Eq. (3) into Eq. (2), we find that the wiggling averaging is nonzero only when $\nu$ is close to an even integer $h=-(2 p \pm 2)[5]$ 
and obtain the equation for even harmonics:

$$
\begin{aligned}
\left(\frac{\partial}{\partial z}+\frac{\nabla_{1}^{2}}{2 i h k_{1}}\right) E_{h}\left(\Delta \nu_{h}, \mathbf{x} ; z\right)= & -i \frac{e K_{h}}{2 \epsilon_{0} \gamma_{0}} e^{i \Delta \nu_{h} k_{u} z} \int \frac{k_{1} d \theta}{2 \pi} e^{-i \nu \theta} \\
& \times \frac{K}{\gamma_{0} k_{u}} \sum_{j=1}^{N_{e}} \delta^{\prime}\left(x-x_{j}^{\beta}\right) \delta\left(y-y_{j}^{\beta}\right) \delta\left(\theta-\theta_{j}\right),
\end{aligned}
$$

where the effective coupling strength is

$$
K_{h}=K(-1)^{(h-2) / 2} J_{h / 2}^{\prime}(h \xi), \quad h=2,4, \ldots
$$

Hence, in the forward $z$ direction of a perfectly aligned undulator trajectory, even harmonic emissions are present due to the transverse gradient of the electron current in the wiggling plane.

The electron distribution in phase space is described by the Klimontovich distribution function $f(\theta, \eta, \mathbf{x}, \mathbf{p} ; z)$, where $\eta=\left(\gamma-\gamma_{0}\right) / \gamma_{0}$, and $\mathbf{p}=d \mathbf{x} / d z$ are the conjugate variables to $\theta$ and $\mathrm{x}$. Using the Pierce parameter $\rho[6]$, we introduce the following scaled variables:

$$
\begin{aligned}
\bar{z} & =2 \rho k_{u} z, \quad \bar{\eta}=\frac{\eta}{\rho}, \quad \bar{\nu}_{h}=\frac{\Delta \nu_{h}}{2 \rho} \\
\overline{\mathbf{x}} & =\mathbf{x} \sqrt{2 k_{1} k_{u} \rho}, \quad \overline{\mathbf{p}}=\mathbf{p} \sqrt{\frac{k_{1}}{2 k_{u} \rho}} \\
a_{h}\left(\bar{\nu}_{h}, \overline{\mathbf{x}} ; \bar{z}\right) & =\frac{-e K_{h}}{4 \gamma_{0}^{2} m c^{2} k_{u} \rho} e^{-i \Delta \nu_{h} k_{u} z} E_{h}\left(\Delta \nu_{h}, \mathbf{x}, z\right) .
\end{aligned}
$$

Equations (5) and ( 7 ) can be written as

$$
\left(\frac{\partial}{\partial \bar{z}}+i \bar{\nu}_{h}+\frac{\bar{\nabla}_{\perp}^{2}}{2 i h}\right) a_{h}=\left(\frac{K_{h}}{K_{1}}\right)^{2} \int d^{2} \bar{p} \int d \bar{\eta} \int \frac{2 \rho d \theta}{2 \pi} e^{-i \nu \theta} \begin{cases}f(\bar{z}) & \text { odd } h \\ \frac{i K}{\gamma_{0} k_{u}} \frac{\partial f}{\partial x} & \text { even } h\end{cases}
$$

The erolution of the distribution function is governed by the Klimontovich 
equation integrated along the unperturbed trajectory [3]:

$$
\begin{aligned}
f(z)=f(0) & +\int_{0}^{\bar{z}} d \bar{s} \sum_{\text {odd } h} \int d\left(\bar{\nu}_{h}\right) e^{i \nu \theta^{(0)}} a_{h}\left(\bar{\nu}_{h}, \overline{\mathbf{x}}^{(0)}, \bar{s}\right) \frac{\partial}{\partial \bar{\eta}} f\left(\theta^{(0)}, \bar{\eta}, \overline{\mathbf{x}}^{(0)}, \overline{\mathbf{p}}^{(0)} ; \bar{s}\right) \\
& +\int_{0}^{\bar{z}} d \bar{s} \sum_{\text {even } h} \int d\left(\bar{\nu}_{h}\right) e^{i \nu \theta^{(0)}} a_{h}\left(\bar{\nu}_{h}, \overline{\mathbf{x}}^{(0)}, \bar{s}\right) \frac{i K}{\gamma_{o} k_{u}} \frac{\partial^{2} f}{\partial x \partial \bar{\eta}}+\text { c.c. } .
\end{aligned}
$$

Here the summation of $h$ is extended to include the interactions with the even harmonics. The unperturbed trajectory is described by

$$
\begin{aligned}
& \theta^{(0)}(s)=\theta+\phi(\bar{s}-\bar{z}) \quad \text { with } \quad \phi=\bar{\eta}-\left(\overline{\mathbf{p}}^{2}+\bar{k}_{\beta}^{2} \overline{\mathbf{x}}^{2}\right) / 2, \\
& \overline{\mathbf{x}}^{(0)}(s)=\overline{\mathbf{x}} \cos \left(\bar{k}_{\beta}(\bar{s}-\bar{z})\right)+\frac{\overline{\mathbf{p}}}{\bar{k}_{\beta}} \sin \left(\bar{k}_{\beta}(\bar{s}-\bar{z})\right) \\
& \overline{\mathbf{p}}^{(0)}(s)=-\bar{k}_{3} \overline{\mathbf{x}} \sin \left(\bar{k}_{\beta}(\bar{s}-\bar{z})\right)+\overline{\mathbf{p}} \cos \left(\bar{k}_{\beta}(\bar{s}-\bar{z})\right)
\end{aligned}
$$

where $\bar{k}_{\beta}=k_{3} /\left(2 k_{u} \rho\right)$ is the scaled betatron focusing strength. $f(0)=f_{0}+\delta f_{0}$ contains the initial fluctuation $\delta f_{0}$ as well as the initial smooth distribution $f_{0}$, which is assumed to be

$$
f_{0}\left(\bar{\eta} \cdot \overline{\mathbf{p}}^{2}+\bar{k}_{\beta}^{2} \overline{\mathbf{x}}^{2}\right)=\frac{1}{2 \pi \bar{\sigma}_{x}^{2} \bar{k}_{\beta}^{2}} \exp \left[-\frac{\left(\overline{\mathbf{p}}^{2}+\bar{k}_{\beta}^{2} \overline{\mathbf{x}}^{2}\right)}{2 \bar{\sigma}_{x}^{2} \bar{k}_{\beta}^{2}}\right] \frac{e^{-\bar{\eta}^{2} /\left(2 \bar{\sigma}_{\eta}^{2}\right)}}{\sqrt{2 \pi} \bar{\sigma}_{\eta}}
$$

where $\bar{\sigma}_{x}=\sigma_{x} \sqrt{2 \rho k_{u} z}$ and $\bar{\sigma}_{\eta}=\sigma_{\eta} / \rho$ are the scaled beam size and scaled energy spread, respectively.

Coherent harmonic radiation is generated through nonlinear harmonic interactions. After a certain stage of exponential growth, the dominant nonlinear term has been shown to be predominantly driven by the fundamental field [3]. Thus, we consider the nonlinear harmonic bunching determined by the fundamental field only. In the small signal regime, we keep the $a_{1}$ term only in Eq. (11) and solve it by iteration:

$$
f(z) \approx f_{0}+\delta f_{0}+\sum_{\text {all } h} f_{h}(z)+\text { c.c. }
$$


where

$$
f_{h}(z)=\left[\prod_{m=1}^{h}\left(\int_{0}^{\bar{s}_{m-1}} d \bar{s}_{m} \int d\left(\bar{\nu}_{m}\right) e^{i\left(1+2 \rho \bar{\nu}_{m}\right) \theta^{(0)}\left(s_{m}\right)} a_{1}\left(\bar{\nu}_{m}, \overline{\mathbf{x}}^{(0)}\left(s_{m}\right) ; \bar{s}_{m}\right) \frac{\partial}{\partial \bar{\eta}}\right)\right] f_{0}
$$

for $h \geq 1$ and $s_{0}=\bar{z}$. We note that Eq. (14) with Eq. (15) is the approximate solution of Eq. (11) when the nonlinear harmonic generation dominates over the linear harmonic generation or the spontaneous harmonic emission [3]. It becomes an increasingly good approximation as the fundamental field is significantly amplified.

The evolution of the fundamental field is obtained by solving Eq. (10) with $h=1$ and $f$ replaced by $\delta f_{0}+f_{1}(z)[3,7]$ :

$$
\begin{aligned}
a_{1}(\bar{\nu}, \overline{\mathbf{x}} ; \bar{z})=e^{-i \mu_{1} \bar{z}} A_{1}(\overline{\mathbf{x}})[ & \int d^{2} \bar{x}^{\prime} A_{1}\left(\overline{\mathbf{x}}^{\prime}\right) a_{1}\left(\bar{\nu}, \overline{\mathbf{x}}^{\prime} ; 0\right)+\int d^{2} \bar{x}^{\prime} \int d^{2} \bar{p} \int d \bar{\eta} \\
& \left.\times \delta f_{0}\left(\bar{\nu}, \overline{\mathbf{x}}^{\prime}, \overline{\mathbf{p}}, \bar{\eta}\right) \int_{-\infty}^{0} d \tau A_{1}\left(\overline{\mathbf{x}}^{(0)}\right) e^{i\left(\phi-\mu_{1}\right) \tau}\right]
\end{aligned}
$$

where $\mu_{1}$ is the complex growth rate of the fundamental mode $A_{1}(\overrightarrow{\mathbf{x}})$ with the largest imaginary part of $\mu_{1}$. The first term of Eq. (16) describes the process of coherent amplification from the initial coherent signal $a_{1}(\bar{\nu}, \overline{\mathbf{x}} ; 0)$, and the second term of Eq. (16) describes the process of self-amplified spontaneous emission from the initial shot noise $\delta f_{0}$. Inserting Eq. (14) into Eq. (10), we find that $a_{h}(h>1)$ is determined by $f_{h}$ with a complex growth rate $h \mu_{1}$, and that the characteristics of the nonlinear harmonic generation are all determined by the fundamental field. While the transverse profile of the odd harmonics is azimuthally symmetric just as the fundmental mode, the transverse profile of the even harmonics possesses the odd symmetry in the wiggling plane (the $x$ plane) due to the transverse gradient effect in Eq. (10). 


\section{Third-Harmonic Radiation}

The most significant nonlinear harmonic generation occurs at the third harmonic, given by Eq. (10) for $h=3$ and $f$ replaced by $f_{3}$ of Eq. (15). For a seeded FEL, we assume that the external signal matches optimal detuning $\bar{\nu}_{0}$ for the fundamental field (with a complex growth rate $\mu_{0}$ that has the maximum imaginary part). We can set $\bar{\nu}_{1}=\bar{\nu}_{0}$ and $\bar{\nu}_{3}=3 \bar{\nu}_{0}$ and drop the frequency dependence of $a_{1}$ and $a_{3}$ in Eq. (10). In view of Eq. (16), we write $a_{1}(\overrightarrow{\mathbf{x}} ; \bar{z})=e^{-i \mu_{0} \bar{z}} A_{1}(\overline{\mathbf{x}})$, where $A_{1} \approx A_{0} e^{-w_{1} R^{2}}$ is the fundamental mode $\left(R=|\overline{\mathbf{x}}| / \bar{\sigma}_{x}=|\mathbf{x}| / \sigma_{x}\right)$, and $A_{0}$ is the appropriate normalization coefficient. Thus, we can write the third nonlinear harmonic $a_{3}(\overline{\mathbf{x}} ; \bar{z})=e^{-3 i \mu_{0} \bar{z}} A_{3}(\overline{\mathbf{x}})$ with the transverse profile $A_{3}$ given by [3]:

$$
\begin{aligned}
& {\left[-3 i\left(\mu_{0}-\bar{\nu}_{0}\right)+\frac{\bar{\nabla}_{\perp}^{2}}{6 i}\right] A_{3}(\overline{\mathbf{x}})=\left(\frac{K_{3}}{K_{1}}\right)^{2} \int d^{2} \bar{p} \int d \bar{\eta}} \\
& \times \int_{-\infty}^{0} d \tau_{1} e^{3 i\left(\phi-\mu_{0}\right) \tau_{1}} A_{1}\left(\overline{\mathbf{x}}^{(0)}\left(s_{1}\right)\right) \frac{\partial}{\partial \bar{\eta}} \int_{-\infty}^{0} d \tau_{2} e^{2 i\left(\phi-\mu_{0}\right) \tau_{2}} \\
& \times A_{1}\left(\overline{\mathbf{x}}^{(0)}\left(s_{2}\right)\right) \frac{\partial}{\partial \bar{\eta}} \int_{-\infty}^{0} d \tau_{3} e^{i\left(\phi-\mu_{0}\right) \tau_{3}} A_{1}\left(\overline{\mathbf{x}}^{(0)}\left(s_{3}\right)\right) \frac{\partial \bar{f}_{0}}{\partial \bar{\eta}}
\end{aligned}
$$

where $\tau_{m}=\bar{s}_{m}-\bar{s}_{m-1}$ for $m=1,2,3$, and $\bar{s}_{0}=\bar{z}$. We have extended the lower limit of the integral $\int d \tau_{m}$ to $-\infty$ due to the exponential growth. Solving Eq. (17) with the Hankel transformation, we obtain [3]

$$
A_{3}(R)=\left(\frac{K_{3}}{K_{1}}\right)^{2} A_{0}^{3} \int_{0}^{\infty} Q d Q J_{0}(Q R) H(Q)
$$

where

$$
\begin{aligned}
H(Q)= & \frac{-1 / u_{1}^{2}}{12\left(\mu_{0}-\bar{\nu}_{0}\right)-2 Q^{2} /\left(3 \bar{\sigma}_{x}^{2}\right)} \int_{-\infty}^{0} d \tau_{1} \int_{-\infty}^{0} d \tau_{2} \int_{-\infty}^{0} d \tau_{3} \frac{3 \tau_{1}\left(3 \tau_{1}+2 \tau_{2}\right)\left(3 \tau_{1}+2 \tau_{2}+\tau_{3}\right)}{U} \\
& \times \exp \left[-\frac{\bar{\sigma}_{n}^{2}}{2}\left(3 \tau_{1}+2 \tau_{2}+\tau_{3}\right)^{2}-i \mu_{0}\left(3 \tau_{1}+2 \tau_{2}+\tau_{3}\right)-\frac{Q^{2}}{4 \omega_{1} S}\right]
\end{aligned}
$$


and

$$
\begin{aligned}
S= & \frac{U}{V+\sum_{m=1,2,3} \sin ^{2}\left(\bar{k}_{\beta} \sum_{l=1}^{m} \tau_{l}\right)}, \\
U= & {\left[\sum_{m=1}^{3} \sin ^{2}\left(\bar{k}_{\beta} \sum_{l=1}^{m} \tau_{l}\right)\right]\left[\sum_{m=1}^{3} \cos ^{2}\left(\bar{k}_{\beta} \sum_{l=1}^{m} \tau_{l}\right)\right] } \\
& -\left[\sum_{m=1}^{3} \sin \left(\bar{k}_{\beta} \sum_{l=1}^{m} \tau_{l}\right) \cos \left(\bar{k}_{\beta} \sum_{l=1}^{m} \tau_{l}\right)\right]^{2}+V^{2}+3 V, \\
V= & \frac{1}{2 w_{1}}+\frac{i}{2 w_{1}} \bar{k}_{\beta}^{2} \bar{\sigma}_{x}^{2}\left(3 \tau_{1}+2 \tau_{2}+\tau_{3}\right) .
\end{aligned}
$$

Equation (18) can be computed using a discrete Hankel transformation. In general, the third-harmonic radiation is also transversely coherent with a Gaussian-like profile and a narrower spot size than the fundamental (see Figs. 1 and 2).

For a SASE FEL, the fundamental radiation starts with a white noise spectrum and has a finite gain bandwidth. In the time domain, the temporal structure of the fundamental is chaotic with many random spikes. Due to the nonlinear generation mechanism, the temporal structure of the third-harmonic radiation is similar to the fundamental, but with more fluctuation from spike to spike. It can be shown that $[3]$

$$
A_{3}(\theta, R ; \bar{z}) \approx\left(\frac{K_{3}}{K_{1}}\right)\left[G_{1}(\theta ; \bar{z})\right]^{3} A_{0}^{3} H_{0} e^{-w_{3} R^{2}}
$$

where $G_{1}(\theta ; \bar{z})$ is a Gaussian random variable in $\theta$ and a slowly varying function in $\bar{z}$ for $\operatorname{SASE}\left(G_{1}=1\right.$ for $\left.\mathrm{CA}\right), H_{0}=\left(K_{3} / K_{1}\right) \int Q d Q H_{3}(Q)$, and $w_{3}$ characterizes the transverse profile of the third-harmonic radiation. The average radiation power can be obtained by integrating over the transverse intensity profile and averaging over the temporal fluctuation. Thus, we have [3]

$$
\left(\frac{P_{3}}{\rho P_{\text {beam }}}\right) \approx\left|H_{0}\right|^{2} \frac{16 w_{l r}^{3}}{w_{3 r}}\left(\frac{P_{1}}{\rho P_{\text {beam }}}\right)^{3} \times \begin{cases}1 & \text { CA } \\ 6 & \text { SASE }\end{cases}
$$


Here $P_{\text {beam }}=2 \pi \sigma_{x}^{2} \vartheta m c^{3} n_{0}$ is the total electron beam power, and $w_{1 r}$ and $w_{3 r}$ are the real parts of $u_{1}$ and $w_{3}$. Thus, the third-harmonic radiation for a SASE FEL has a power level roughly 6 times larger than the corresponding steady state case, but with more shot-to-shot fluctuations compared to the fundamental [3]. The third-harmonic bunching parameter is obtained by averaging $\left(e^{-3 i \theta} f_{3}\right)$ over the 6 -D phase-space volume and taking the absolute value [3]:

$$
\begin{aligned}
b_{3} & =8 u_{1 r}^{3 / 2}\left(\frac{P_{1}}{\rho P_{\text {beam }}}\right)^{3 / 2} \mid \int_{-\infty}^{0} d \tau_{1} \int_{-\infty}^{0} d \tau_{2} \int_{-\infty}^{0} d \tau_{3} \frac{3 \tau_{1}\left(3 \tau_{1}+2 \tau_{2}\right)\left(3 \tau_{1}+2 \tau_{2}+\tau_{3}\right)}{4 w_{1}^{2} U} \\
& \times \exp \left[-\frac{\bar{\sigma}_{\eta}^{2}}{2}\left(3 \tau_{1}+2 \tau_{2}+\tau_{3}\right)^{2}-i \mu_{0}\left(3 \tau_{1}+2 \tau_{2}+\tau_{3}\right)\right] \mid \times \begin{cases}1 & \text { CA, } \\
1.3 & \text { SASE }\end{cases}
\end{aligned}
$$

For example. using the design parameters (see Table 1) for the low-energy undulator test line (LEUTL) FEL at the Advanced Photon Source [8] and the proposed Linac Coherent Light Source (LCLS) at Stanford Linear Accelerator Center [9], we compute the transverse profile of the third harmonic and plot it in comparison with the fundamental profile in Figs. 1 and 2. The thirdharmonic power and the bunching parameter are also calculated according to Eqs. (22) and (23). Table 1 lists the results when the fundamental power reaches one half of the saturation power, when the exponential growth process is supposed to stop. We have compared the evolution of the third-harmonic

power for the LEUTL FEL with the steady-state MEDUSA simulation [2], and the third-harmonic bunching for the LCLS case with the steady-state GINGER simulation [3]. Good agreement for both cases have been found.

\section{Second-Harmonic Radiation}

The second-harmonic radiation can be calculated from Eq. (10) with $h=2$ and $f$ replaced by $f_{2}$ of Eq. (15). One can follow the same procedure as in Sec. 
3 to solve for the second-harmonic field for CA and SASE, except that the Hankel transformation should be replaced by the 2-D Fourier transformation in $x$ and $y$ because the radiation profile has the odd symmetry in $x$. Since the wiggling amplitude $K /\left(\gamma_{0} k_{u}\right)$ is usually much smaller than the rms beam size $\sigma_{x}$, the power of the second-harmonic radiation is less than that of the third harmonic. We can estimate the power of the second-harmonic radiation by

$$
\frac{P_{2}}{b_{2}^{2}} \approx\left(\frac{K}{\gamma_{0} k_{u} \sigma_{x}}\right)^{2}\left(\frac{K_{2}}{K_{3}}\right)^{2} \frac{P_{3}}{b_{3}^{2}} .
$$

Here the second harmonic bunching parameter $b_{2}$ is obtained by averaging $\left(e^{-2 i \theta} f_{2}\right)$ over the 6 -D phase-space volume and taking the absolute value

$$
\begin{aligned}
b_{2}= & 4 w_{1 r}\left(\frac{P_{1}}{\rho P_{\text {beam }}}\right) \mid \int_{-\infty}^{0} d \tau_{1} \int_{-\infty}^{0} d \tau_{2} \frac{2 \tau_{1}\left(2 \tau_{1}+\tau_{2}\right)}{4 w_{1}^{2} U_{2}} \\
& \times \exp \left[-\frac{\bar{\sigma}_{\eta}^{2}}{2}\left(2 \tau_{1}+\tau_{2}\right)^{2}-i \mu_{0}\left(2 \tau_{1}+\tau_{2}\right)\right] \mid
\end{aligned}
$$

where

$$
\begin{aligned}
U_{2}= & {\left[\sum_{m=1}^{2} \sin ^{2}\left(\bar{k}_{\beta} \sum_{l=1}^{m} \tau_{l}\right)\right]\left[\sum_{m=1}^{2} \cos ^{2}\left(\bar{k}_{\beta} \sum_{l=1}^{m} \tau_{l}\right)\right] } \\
& -\left[\sum_{m=1}^{2} \sin \left(\bar{k}_{\beta} \sum_{l=1}^{m} \tau_{l}\right) \cos \left(\bar{k}_{\beta} \sum_{l=1}^{m} \tau_{l}\right)\right]^{2}+V_{2}^{2}+2 V_{2}, \\
V_{2}= & \frac{1}{2 w_{1}}+\frac{i}{2 w_{1}} \bar{k}_{\beta}^{2} \bar{\sigma}_{x}^{2}\left(2 \tau_{1}+\tau_{2}\right) .
\end{aligned}
$$

Using the LEUTL and LCLS examples, we calculate the second-harmonic bunching and estimate the second-harmonic power by Eq. (24). From Table 1 , we see that a significant amount of second-harmonic radiation can be generated in the LEUTL FEL because the wiggling amplitude (proportional to $\left.1 / \gamma_{0}\right)$ is about one-third of the beam size. However, for $\mathrm{x}$-ray FELs employing a high-energy electron beam, such as the LCLS, the second-harmonic radiation is much reduced. 


\section{Conclusion}

We have presented a perturbation scheme to analyze the 3-D evolution of the nonlinear harmonic radiation in coherent amplification and self-amplified spontaneous emission, with explicit calculation of second-harmonic and thirdharmonic radiation based on current high-gain FEL projects. The transverse coherence and the substantial power level of the third harmonic could be useful in extending the short wavelength reach of a high-gain FEL.

We thank S. Biedron, Y. Chae, W. Fawley, H. Freund, S. Milton, and C. Pelligrini for useful discussions. This work was supported by the U. S. Department of Energy; Office of Basic Energy Sciences, under Contract No. W-31-109ENG-38.

\section{References}

[1] R. Bonifacio, L. De Salvo, and P. Pierini, Nucl. Instr. Meth. A 293, 627 (1990).

[2] H.P. Freund. S.G. Biedron, and S.V. Milton, IEEE J. Quantum Electron. QE-36, 275 (2000); Nucl. Instr. Meth. A 445, 53 (2000).

[3] Z. Huang and K.-J. Kim, submitted to Physical Review E.

[4] W.B. Colson, IEEE J. Quantum Electron. QE-17, 1417 (1981).

[5] M.J. Schmitt and C.J. Elliott, Phys. Rev. A 34, 4843 (1986); Phys. Rev. A 41, 3853 (1990); Nucl. Instr. Meth. A 296, 394 (1990).

[6] R. Bonifacio, C. Pellegrini, and L.M. Narducci, Opt. Comm. 50, 373 (1984).

[7] Z. Huang and K.-J. Kim, in these proceedings.

[8] S.V. Milton et al., Nucl. Instr. Meth. A 407, 210 (1998).

[9] Linac Coherent Light Source Design Study Report, SLAC-R-521, 1998. 


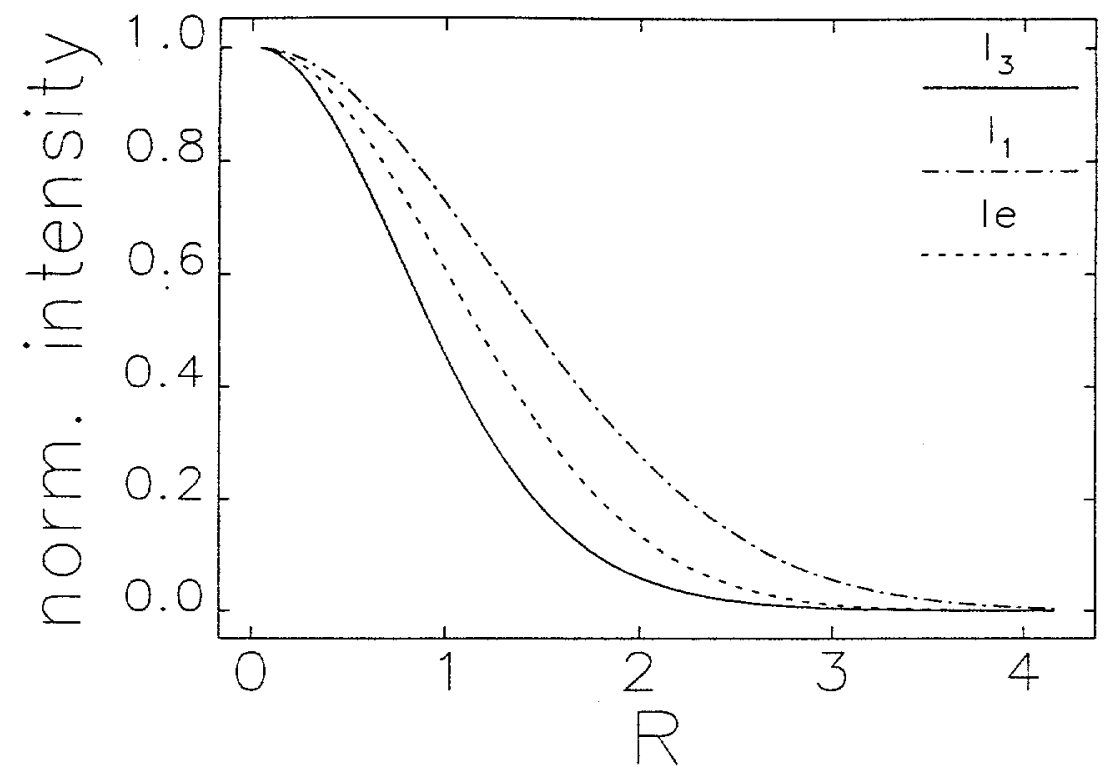

Fig. 1. Transverse profiles of the LEUTL third harmonic $\left(I_{3}\right)$, the fundamental $\left(I_{1}\right)$, and the electron beam $\left(I_{e}\right)$ as functions of the transverse position in units of the electron beam size.

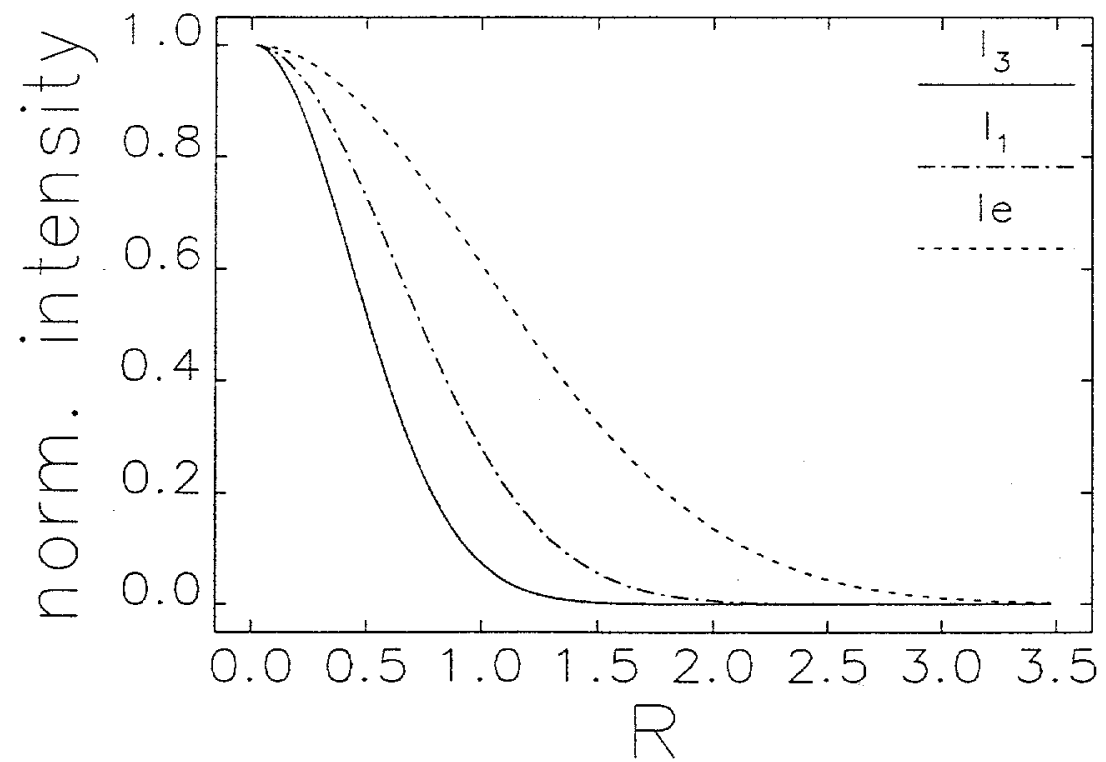

Fig. 2. Transverse profiles of the LCLS third harmonic $\left(I_{3}\right)$, the fundamental $\left(I_{1}\right)$, and the electron beam $\left(I_{e}\right)$ as functions of the transverse position in units of the electron beam size. 
Table 1

Nonlinear Harmonic Generation for SASE FELS

\begin{tabular}{|lcc|}
\hline & LEUTL & LCLS \\
E-beam and Undulator & & \\
energy & $220 \mathrm{MeV}$ & $14.4 \mathrm{GeV}$ \\
peak current & $150 \mathrm{~A}$ & $3400 \mathrm{~A}$ \\
normalized emittance & $5 \mu \mathrm{m}$ & $1.5 \mu \mathrm{m}$ \\
energy spread & $0.1 \%$ & $0.02 \%$ \\
average beta function & $1.5 \mathrm{~m}$ & $18 \mathrm{~m}$ \\
undulator period & $3.3 \mathrm{~cm}$ & $3 \mathrm{~cm}$ \\
undulator strength & 3.1 & 3.71 \\
Fundamental Radiation & & \\
resonant wavelength & $518 \mathrm{~nm}$ & $1.5 \AA$ \\
power gain length & $0.67 \mathrm{~m}$ & $6.1 \mathrm{~m}$ \\
saturation power $\left(P_{\text {sat }}\right)$ & $70 \mathrm{MW}$ & $8 \mathrm{GW}$ \\
Harmonics at $P_{1}=P_{\text {sat }} / 2$ & & \\
$3^{\text {rd }}$-harmonic power & $3.6 \mathrm{MW}$ & $15 \mathrm{MW}$ \\
$3^{\text {rd }}$-harmonic bunching & 0.39 & 0.018 \\
$2^{\text {nd }}$-harmonic power & $550 \mathrm{~kW}$ & $15 \mathrm{~kW}$ \\
$2^{\text {nd }}$-harmonic bunching & 0.47 & 0.056 \\
\hline
\end{tabular}

